\title{
Ustekinumab: an evidence-based review of its effectiveness in the treatment of psoriasis
}

This article was published in the following Dove Press journal:

Core Evidence

3 July 2010

Number of times this article has been viewed

\section{Eliana Krulig \\ Kenneth B Gordon}

NorthShore University HealthSystem, University of Chicago, Pritzker School of Medicine, Skokie, IL, USA
Correspondence: Kenneth B Gordon, MD Head of Dermatology NorthShore University HealthSystem, Associate Professor of Dermatology, University of Chicago, Pritzker School of Medicine, 9933 Woods Dr Skokie, IL 60077, USA $\mathrm{Tel}+\mathrm{I}$ (847) 663-8539 Fax + I (847) 663-8536 Email kgordon@northshore.org
Introduction: Psoriasis is a chronic inflammatory skin disease affecting approximately $2 \%$ to $3 \%$ of the population worldwide. Discoveries over the past 3 to 5 years have significantly altered our view of psoriasis as primarily a T-cell mediated condition. The most recent research has demonstrated the essential role of specific cytokines in the development of this complex disease, including TNF- $\alpha$, interleukin-23 (IL-23), and potentially, IL-22. These are all part of a newly defined autoimmune pathway directed by specialized T cells called Th17 helper T cells. Ustekinumab is a fully human monoclonal antibody that targets IL-12 and IL-23, thus targeting both Th1 and Th17 arms of immunity. It has a promising efficacy and safety profile that not only represents a valuable treatment alternative, but also a continuation in our constantly evolving understanding of this disorder.

Aims: To review the emerging evidence supporting the use of ustekinumab in the management of moderate to severe plaque psoriasis.

Evidence review: There is clear evidence that ustekinumab is effective in the treatment of moderate to severe psoriasis. Phase III trials (PHOENIX 1 and 2) demonstrated a statistically significant difference between Psoriasis Area and Severity Index (PASI) 75 responses achieved by patients receiving ustekinumab, given as a $45 \mathrm{mg}$ or $90 \mathrm{mg}$ subcutaneous injection every 12 weeks, than their placebo counterparts. Treatment with this novel agent resulted in a rapid onset of action, with over $60 \%$ of treated patients attaining Physician's Global Assessment (PGA) scores of "cleared" or "minimal" by week 12. Quality of life assessments paralleled clinical improvements.

Clinical potential: Ustekinumab is an effective and efficient therapeutic option for patients with moderate to severe psoriasis. Although further studies are required to establish ustekinumab's place in the therapy of psoriasis, with its convenient dosing schedule and rapid onset of action, this drug could provide a great addition to the current therapeutic armamentarium available for psoriatic patients.

Keywords: ustekinumab, psoriasis, biologics, interleukin-12 (IL-12), interleukin-23 (IL-23), IL-12/23, evidence

Core evidence outcomes summary for ustekinumab in psoriasis

\begin{tabular}{lll}
$\begin{array}{l}\text { Outcome } \\
\text { measure }\end{array}$ & Evidence & Implications \\
\hline $\begin{array}{l}\text { Disease-oriented evidence } \\
\text { Statistically significant }\end{array}$ & Clear & $\begin{array}{l}\text { Ustekinumab effectively controls psoriasis } \\
\text { and improves clearance rates compared } \\
\text { to placebo } 75 \text { responses }\end{array}$ \\
\end{tabular}

submit your manuscript $\mid$ www.dovepress.com 


\begin{tabular}{|lll|}
\hline $\begin{array}{l}\text { (Continued) } \\
\text { Outcome } \\
\text { measure }\end{array}$ & Evidence & Implications \\
\hline Time to relapse & Substantial & $\begin{array}{l}\text { The median time to loss of PASI } 75 \text { after } \\
\text { withdrawal was about I5 weeks }\end{array}$ \\
$\begin{array}{l}\text { Patient-oriented evidence } \\
\begin{array}{l}\text { Quality of life } \\
\text { improvement }\end{array}\end{array}$ & Clear & $\begin{array}{l}\text { Ustekinumab considerably improves } \\
\text { quality of life in psoriatic patients } \\
\text { compared to placebo }\end{array}$ \\
Tolerability & Moderate & $\begin{array}{l}\text { Safety profile similar to other biologics. } \\
\text { Further studies are required to evaluate }\end{array}$ \\
& & long-term tolerability \\
Economic evidence & & \\
Cost effectiveness & No evidence & \\
\hline Abbreviations: PASI, Psoriasis Area and Severity Index. & \\
\hline
\end{tabular}

\section{Scope, aims, and objectives}

Ustekinumab (Stelara ${ }^{\circledR}$; Centocor, Inc), previously referred to as CNTO 1275, is a fully human monoclonal antibody that targets interleukin-12 (IL-12) and interleukin-23 (IL-23). It binds with high affinity to their shared p40 subunit, neutralizing their bioactivity by blocking interactions with their cognate receptors. This review seeks to assess the emerging evidence for ustekinumab in the management of psoriasis.

\section{Methods}

English language literature searches were conducted on January 1st, 2009 in the following databases, searching from the beginning of the database to current date unless otherwise stated. The search strategy was "ustekinumab AND psoriasis" and "CNTO 1275 AND psoriasis":

- PubMed, http://www.ncbi.nlm.nih.gov/pubmed. Limits imposed for specificity: "English," "clinical trial," "meta analysis," "randomized controlled trial," "humans"

- EMBASE, http://www.embase.com

Table I Evidence base included in the review

\begin{tabular}{lll}
\hline Category & Number of records \\
\cline { 2 - 3 } & Full papers & Abstracts \\
\hline Initial search & 3 & 0 \\
Additional papers identified & I (comment) & 0 \\
Search update, new records & I & 0 \\
Level I clinical evidence & 0 & 0 \\
Level 2 & 4 & 0 \\
Level $\geq 3$ & 0 & 0 \\
Economic evidence & 0 & 0 \\
Total records included & 4 & 0 \\
\hline
\end{tabular}

- National Guideline Clearinghouse, http://www. guideline.gov

- Cochrane Database of Systematic Reviews (CDSR), http://www.cochrane.org

Following the search, a total of three full papers were included in the evidence base, one phase II trial and two phase III trials. The search on PubMed was updated on June 1st, 2009. One new record was identified and included in the review (a phase II trial). The selected records are summarized in Table 1.

\section{Disease overview}

Psoriasis is a chronic inflammatory skin disease affecting approximately $2 \%$ to $3 \%$ of the population worldwide. ${ }^{1,2}$ Characterized by well-demarcated itchy red, scaly plaques, it can present with a wide clinical variability. Plaque-type is the most common form (and the one we will refer to throughout

Table 2 Established treatment options for psoriasis

\begin{tabular}{ll}
\hline Category & Treatment \\
\hline Topical & Corticosteroids \\
& Vitamin D analogs \\
& Tars \\
& Others (anthralin, salicylic acid, retinoids) \\
& \\
Systemic & UVB \\
Phototherapy & PUVA \\
Conventional & Methotrexate \\
& Cyclosporine A \\
& Acitretin \\
Biologics & Alefacept \\
& Adalimumab \\
& Etanercept \\
& Infliximab \\
\hline
\end{tabular}


this review), but other forms include guttate, pustular, and erythrodermic.

Discoveries over the past 3 to 5 years have significantly altered our view of psoriasis as primarily a Th1 mediated condition. The most recent research has demonstrated the essential role of specific cytokines in the development of this complex disease, including tumor necrosis factor alpha (TNF- $\alpha$ ), IL-23, and, potentially, IL-22. These are all part of a newly defined autoimmune pathway directed by specialized T cells called Th17 helper T cells. ${ }^{3,4}$

This multisystemic disorder, primarily affecting the skin and joints (psoriatic arthritis) is associated with several comorbidities, including inflammatory bowel disease, lymphoma, coronary heart disease, obesity, and metabolic syndrome. ${ }^{5-8}$ There is a significant emotional burden on these individuals, and psychiatric/psychological comorbidities such as depression, suicidal ideation, and other mood disorders, have also been linked..$^{9-12}$ The negative impact of psoriasis on patients is comparable to that of heart disease, cancer, and arthritis. ${ }^{13,14}$

The cause of psoriasis remains unknown. However, linkages to various genetic traits have been identified, as well as a myriad of environmental factors that trigger/exacerbate the condition. ${ }^{15-17}$ Our knowledge on the pathophysiology of psoriasis has been furthered deepened during the last decade. Previously considered a disorder of keratinocyte hyperproliferation, it is now understood that its clinical manifestations are secondary to a complex interplay between immune cells, skin cells and dermal endothelium, and the interactions of their pro-inflammatory cytokines, chemokines and various chemical mediators. ${ }^{18}$

The new psoriasis model is explained by the interaction between epidermal keratinocytes and various immunocytes, including the four different CD4+ subsets: Th1, Th2, Th17, and T-regs. ${ }^{18}$ Recently discovered Th17 cells form part of a pathway mainly regulated by IL-23 (among several cytokines), and have been associated with regulation of autoimmunity. ${ }^{3}$ Although initial experiments were targeted towards IL-12's p40 subunit, serendipitous findings suggested that IL-23 (which shares a common p40 subunit with IL-12) was more important in the psoriasis immunopathogenesis, shifting the paradigm to a Th1/Th17 hypothesis. ${ }^{19,20}$

In the cytokine model (Figure 1), sentinel cells in symptomless skin start producing IL-23 secondary to an unknown stimulus, and possible $\mathrm{TNF}^{21}$ It is important to highlight that these sentinel cells, previously considered to be macrophages and resident dermal dendritic cells (DCs), are currently considered by many investigators to be a different population of DCs that appear during inflammation, called "inflammatory" dermal DCs. This subset, characterized by CD1 $1 \mathrm{c}^{+}$and $\mathrm{CD} 1 \mathrm{c} / \mathrm{BCDA}^{-}{ }^{-}$, are increased significantly in psoriatic skin in comparison to the other dermal DCs populations, and they are thought to contain an even more specialized DCs subset that has the ability to produce mediators like TNF and intracellular nitric oxide synthase. ${ }^{22}$ The later have been termed TIP-DCs (TNF and inducible nitric oxide synthase producing DCs), and are held accountable for the production of IL-23. ${ }^{23}$

In turn, IL-23 sustains Th17 proliferation, hence primarily increasing levels of IL-17 and IL-22 produced by these activated Th17 cells (It has been suggested that Th0 differentiation into Th17 is originally initiated by IL- 6 and TGF- $\beta$, and then supported by IL-23 and TNF- $\alpha$ ). Further downstream, IL-20 and IL-22 have the ability to induce keratinocyte proliferation and epidermal remodeling through phosphorylation of Stat3, a transcription factor implicated in psoriasis. ${ }^{19,24}$ In contrast, IL-12 is associated with the Th1 arm. Through it, IL-12 promotes proliferation of IFN- $\gamma$-producing Th1 cells, which have been associated with regulation of cell-mediated immunity. Th1 cells also secrete TNF- $\alpha$, further contributing to inflammation and development of psoriatic plaques. ${ }^{18,25,26}$

Additional data support the role of IL-12 and IL-23 in psoriasis, when genetic studies found associations between certain polymorphisms of the IL-12 receptor (IL-12R) and IL-23R genes and psoriasis. ${ }^{27}$ It is noteworthy that the sequences/processes depicted above portray a simplified version of what is rather a very complex and intricate model.

Different parameters have been used to estimate the severity of the disease, as well as to evaluate clinical outcomes of therapeutic agents. ${ }^{28}$ Among the most widely accepted, and for the purposes of this review, we will list the Psoriasis Area and Severity Index (PASI), Physician's Global Assessment (PGA), Total Body Surface Area (TBSA), and the Dermatology Life Quality Index (DLQI). The PASI is currently regarded as one of the most reliable and consistent outcome measures, thus is commonly used in clinical studies. ${ }^{29}$ It is a composite index, ranging from 0 to 72 , that assigns a score to the three main characteristics of a psoriatic plaque (erythema, thickness and scaling, scored from 0 to 4 ), weighted by its extension on the four main body areas (head, trunk, upper extremities, and lower extremities). Higher PASI scores are associated with more severe disease. ${ }^{30}$ Improvements in PASI of 50\%, 75\%, and 90\% (PASI 50, 75, and 90, respectively) are often used as clinical outcomes.

Traditionally, the PGA is used in the clinical practice. This method classifies the psoriasis as clear (0), minimal (1), mild (2), moderate (3), severe (4), or very severe (5), depending 

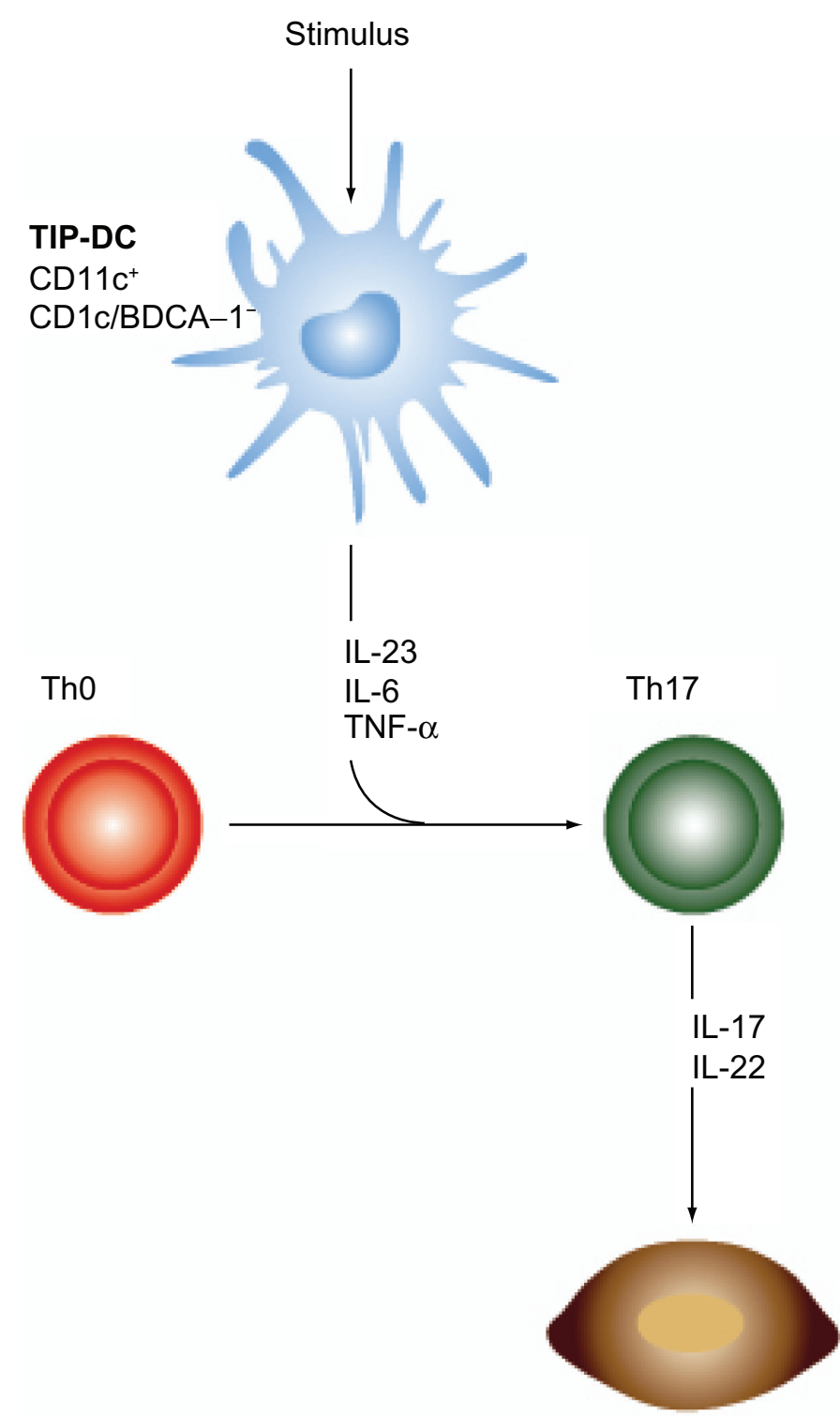

Stat3 active keratinocytes

Keratinocyte hyperproliferation and epidermal thickening

Figure I ThI7 pathway.TIP-dendritic cells (TIP-DCs), secondary to a stimulus, produce IL-23 andTNF- $\alpha$, which sustain differentiation of Th precursors into Th I7 cells (originally initiated by IL-6 and TGF- $\beta$ ). As a result, activated Th 17 cells secrete IL- 17 and IL-22, resulting in keratinocyte hyperproliferation and plaque formation. ${ }^{19}$

on the quality and extension of the psoriatic lesions. TBSA ranges from 0 to $100 \%$ depending on the surface affected. The DLQI, a patient-reported outcome, is a 10-item questionnaire completed by the patients that measures the impact of psoriasis on their quality of life. It can go from 0 to 30 , with higher scores indicative of poor quality of life. ${ }^{29}$

An integral assessment is necessary to label with accuracy the severity of the psoriasis in each individual, and therefore select the appropriate treatment modality based on this result.
By joining physician-reported and patient-reported outcomes, the clinician might estimate the actual toll of psoriasis, not only in their patient's body, but also on their psyche.

\section{Current therapy options}

Psoriasis management depends on various factors, such as clinical variant, extension, severity, presence of psoriatic arthritis, and comorbidities; ranging from local modalities up to systemic regimens. ${ }^{31}$ All the available options are directed 
towards sign/symptom relief, and none of them represent a cure for this chronic illness. A list of established treatment options for psoriasis is shown in Table 2.

The most overall used treatments are topical corticosteroids, which are broadly accessible as mild over-the-counter and much stronger prescribed preparations. ${ }^{32}$ Very efficacious and safe, their use is appropriate for localized disease, but is limited by convenience (eg, frequency of application, messiness, greasy feeling, etc), long-term use side effects (eg, skin atrophy), and impracticality for more than localized disease. ${ }^{33} \mathrm{~A}$ recent study by Strowd et al revealed that from 1986 to 2005, there were an estimated 23.9 million visits for psoriasis management in the US, including both dermatologists and other physicians' visits. During the 20-year period, topical corticosteroids were the most commonly prescribed psoriasis medication, with more potent versions prescribed in recent years. Other popular topical alternatives include vitamin-D derivatives (calcipotriene), tars, anthralin, salicylic acid, and retinoids. Adherence to these topicals has been challenged by similar factors as corticosteroids. ${ }^{33}$

Moderate to severe psoriasis requires systemic interventions. Phototherapy options include broadband and narrowband UVB, and PUVA. NBUVB is a safe and costeffective option. PUVA (oral or topical methoxypsoralens followed by UVA irradiation) is more effective than NBUVB, but long-term use is associated with photoaging, PUVAlentigines, and increased risk of nonmelanoma skin cancer. ${ }^{34}$ Oral psoralen is contraindicated in pregnancy.

Systemic modalities can be further categorized as "conventional" and "biologic". Conventional treatments include methotrexate (MTX), cyclosporine A (CyA), and acitretin. Due to its clinical effects in skin and joints, MTX was the long-standing gold standard systemic agent, but is hepatotoxic, teratogenic, and has many drug interactions. ${ }^{31,35}$ Traditionally, a liver biopsy is required after $1.5 \mathrm{~g}$ cumulative dose. CyA is fast acting and efficacious, but best used in 3- to 4-month courses, due to its many drug interactions and association with nephrotoxicity, hypertension, and possibly lymphoma. Acitretin, a retinoid, differs from MTX and CyA by not immunosuppressing the individuals; however its teratogenic potential considerably limits the use in female of childbearing potential. Often ineffective as monotherapy, is frequently combined with phototherapy. Other side effects of acitretin include dyslipidemia and mucocutaneous irritation. Hydroxyurea, 6-thioguanine, leflunamide, and sulfasalazine are minimally effective, have appreciable toxicity, and are not considered first-line agents. ${ }^{35}$
Aside from ustekinumab, 4 biologic agents are currently FDA approved for psoriasis: alefacept (T-cell modulator), adalimumab, etanercept, and infliximab (TNF- $\alpha$ inhibitors). The TNF- $\alpha$ inhibitors are considered much more effective for skin involvement (better PASI 75 rates), as well as valued for their helpful role in psoriatic arthritis. ${ }^{16,36}$ Efalizumab, a T-cell modulator previously approved for the treatment of psoriasis, was recently withdrawn from the market because of concerns of progressive multifocal leukoencephalopathy (PML) after 4 cases were reported. ${ }^{37} \mathrm{~A}$ brief summary that highlights key points of these agents is depicted in Table 3.

It is important to note that combination, rotational, and sequential therapies are frequent approaches in the treatment of psoriasis. ${ }^{2}$ Combining agents allows the treating physician not only to exploit synergistic effects, but also avoid otherwise toxic doses necessaries to achieve clearance in monotherapeutic regimens. ${ }^{38}$ Even though single-agent therapies may have lower costs and better chance for compliance, more often than not, a single modality will not be sufficient to reach all expectations (such as complete skin clearance, psoriatic arthritis control when applicable, improved quality of life, low rate of adverse events). ${ }^{38}$

\section{Unmet needs}

Current therapeutic options offer patients with moderate to severe psoriasis the opportunity to achieve reasonable clinical outcomes. ${ }^{39}$ Nevertheless, standard treatments are associated with long-term toxicity, side effects, and inconvenience, and biologics are fairly new, hence more time is essential to support the current evidence on efficacy and safety. ${ }^{39,40}$

Studies have yet to determine whether newer treatments satisfy patients' needs. Future agents should meet various specifications in order to increase adherence, and consequently, total psoriasis control. A more convenient administration schedule/route, enhanced long-term efficacy and safety profiles, cost-effectiveness, and tolerability, are among the desirable characteristics that future agents must fulfill. In many cases, efficacy in psoriatic arthritis and nail psoriasis is also desired.

\section{Clinical evidence with ustekinumab in psoriasis}

Ustekinumab (Stelara ${ }^{\circledR}$; Centocor, Inc) is a fully human monoclonal antibody targeting IL-12/23. It does so by binding to their shared p40 subunit, therefore blocking the interaction with their cognate receptors. ${ }^{41}$ This agent thus targets Th1 and Th17 arms of immunity, both of which we now know 
Table 3 Key points of biologic agents approved for the treatment of psoriasis ${ }^{49-57}$

\begin{tabular}{|c|c|c|c|c|c|}
\hline Biologic & Mechanism of action & Administration & $\begin{array}{l}\text { Efficacy as PASI } 75 \\
\text { (phase III trials) }\end{array}$ & Particular safety issues & Comment \\
\hline Alefacept & $\begin{array}{l}\text { Recombinant fusion } \\
\text { protein. Inhibits CD2 } \\
\text { from interacting with } \\
\text { LFA-3 }\end{array}$ & $\begin{array}{l}\text { I5 mg IM qw for } \\
\text { I2 weeks, stop } \\
\text { I2 weeks, restart } \\
\text { another } 12 \text { weeks }\end{array}$ & $\begin{array}{l}\text { At week }|4,2| \% \\
\left(\text { Lebwohl et a }\left.\right|^{51}\right)\end{array}$ & $\begin{array}{l}\text { Lymphopenia (decrease } \\
\text { in CD4 count) }\end{array}$ & $\begin{array}{l}\text { Low rate of responders. } \\
\text { Patients who do } \\
\text { respond, enjoy a long- } \\
\text { term psoriasis remission } \\
\text { and one of the best } \\
\text { safety profiles among } \\
\text { biologics }\end{array}$ \\
\hline Adalimumab & $\begin{array}{l}\text { Fully human monoclonal } \\
\text { antibody. Binds soluble } \\
\text { and transmembrane } \\
\text { TNF- } \alpha\end{array}$ & $\begin{array}{l}80 \mathrm{mg} \text { SC loading } \\
\text { dose, then } 40 \mathrm{mg} \\
\text { SC qow }\end{array}$ & $\begin{array}{l}\text { At week } 16,71 \% \\
\left(\text { Menter et } \mathrm{al}^{53}\right)\end{array}$ & $\begin{array}{l}\text { Injection site reactions, } \\
\text { reactivation of TB, } \\
\text { demyelinating disorders, } \\
\text { and contraindicated } \\
\text { in CHF }\end{array}$ & $\begin{array}{l}\text { Balance between efficacy } \\
\text { and safety. Convenient } \\
\text { dosing schedule }\end{array}$ \\
\hline Etanercept & $\begin{array}{l}\text { Receptor antibody fusion } \\
\text { protein. Binds soluble } \\
\text { TNF- } \alpha \text { and lymphotoxin }\end{array}$ & $\begin{array}{l}50 \mathrm{mg} \mathrm{SC} \text { biw for } \\
\text { I } 2 \text { weeks, followed by } \\
50 \mathrm{mg} \mathrm{qw}\end{array}$ & $\begin{array}{l}\text { At week } 12,49 \% \\
\left(\text { Papp et } \mathrm{a}^{\mathrm{I}^{54}}\right)\end{array}$ & $\begin{array}{l}\text { Injection site reactions, } \\
\text { reactivation of TB, } \\
\text { demyelinating disorders, } \\
\text { and contraindicated } \\
\text { in CHF }\end{array}$ & $\begin{array}{l}\text { Balance between efficacy } \\
\text { and safety. Has been } \\
\text { evaluated for pediatric } \\
\text { psoriasis }\end{array}$ \\
\hline Infliximab & $\begin{array}{l}\text { Chimeric monoclonal } \\
\text { antibody. Binds soluble } \\
\text { and transmembrane } \\
\text { TNF- } \alpha\end{array}$ & $\begin{array}{l}5 \mathrm{mg} / \mathrm{kg} \text { IV at weeks } \\
0,2,6 \text {, and followed } \\
\text { by q8w }\end{array}$ & $\begin{array}{l}\text { At week } 10,80 \% \\
\left(\text { Reich et } \mathrm{a}^{56}\right)\end{array}$ & $\begin{array}{l}\text { Infusion reactions, } \\
\text { reactivation of TB, } \\
\text { demyelinating disorders, } \\
\text { and contraindicated } \\
\text { in CHF }\end{array}$ & $\begin{array}{l}\text { Rapid disease control. } \\
\text { Used for unstable } \\
\text { conditions such as } \\
\text { erythrodermic or } \\
\text { pustular psoriasis }\end{array}$ \\
\hline
\end{tabular}

Abbreviations: PASI, Psoriasis Area and Severity Index; IM, intramuscular; SC, subcutaneous; IV, intravenous; qw, once weekly; qow, every other week; biw, twice weekly; q8w, every 8 weeks. TB, tuberculosis; CHF, congestive heart failure.

are key parts in the immunopathogenesis of psoriasis. It is administered as a subcutaneous injection.

A Biologic License Application (BLA) for ustekinumab was accepted by the US Food and Drug Administration (FDA) in February 2008. In June 2008, during an FDA advisory committee meeting, an expert panel unanimously recommended approval, but extended the review period until December due to concerns of the long-term safety data. On December 2008, the FDA issued a Complete Response letter for ustekinumab's BLA requesting additional information, which was then followed on May 2009 by a 3-month extension in the review timeline in order to establish the product's shelf life. Ustekinumab was approved by the FDA for its use in the United States for moderate-tosevere psoriatic patients in September 2009. It has also been approved in Canada and Europe for the same indication and currently is also being evaluated for Crohn's disease and psoriatic arthritis.

\section{Efficacy}

Phase II data

In an initial phase II trial evaluating the safety and efficacy of single and multiple doses of ustekinumab, 320 patients with moderate-to-severe plaque psoriasis underwent randomization to receive ustekinumab or placebo. ${ }^{41}$ Participants in this double-blind, placebo-controlled, crossover trial were randomly assigned to 5 groups, 64 patients each, receiving placebo or 1 of 4 doses of ustekinumab (one $45 \mathrm{mg}$ dose, one $90 \mathrm{mg}$ dose, four weekly $45 \mathrm{mg}$ doses, or four weekly $90 \mathrm{mg}$ doses). At week 16, patients in the active treatment group with a $\mathrm{PGA} \geq 3$ received one additional injection of their originally assigned dose, and at week 20 , patients in the placebo group crossed over to receive one $90 \mathrm{mg}$ dose of the study drug. No additional or maintenance therapy was given.

Efficacy assessed at week 12 (primary endpoint) revealed statistically significant PASI 75 scores for all groups, achieving $52 \%(45 \mathrm{mg} \times 1), 59 \%(90 \mathrm{mg} \times 1), 67 \%(45 \mathrm{mg} \times 4)$ and $81 \%(90 \mathrm{mg} \times 4)$ in the ustekinumab group, as compared with $2 \%$ in the control group. Responses were maintained through week 24 and then started deteriorating. Clinical responses were supported by the substantial improvements observed in the DLQI in all treatment groups. Efficacy in those patients who crossed over to the treatment group at week 20 to receive one $90 \mathrm{mg}$ dose mirrored the improvements observed in individuals originally assigned to the one $90 \mathrm{mg}$ dose at baseline. Serious adverse events were not statistically different from control. 
All the data suggested a dose and/or schedule dependency that needed to be further explored. Consequently, the success of this trial paved the way for the development of phase III trials evaluating the therapeutic potential of ustekinumab for psoriasis.

\section{Phase III data}

PHOENIX 1 and PHOENIX 2 were the parallel studies designed for this purpose. Both multicenter, randomized, double-blind, placebo-controlled phase III trials shared similar objectives and methods, and elicited comparable efficacy and safety data. Analysis was done using non-responder imputation (NRI). For the purpose of this evidence-based review, data retrieved from these studies will be analyzed independently.

Eligibility criteria for both phase III trials were very similar to the ones described for their phase II predecessor. Men and women aged 18 years or older were eligible if they had a diagnosis of plaque psoriasis for at least 6 months, a baseline PASI score of 12 or higher, at least $10 \%$ body surface area involvement, and were candidates for phototherapy or systemic therapy. Patients were ineligible if they had nonplaque forms of psoriasis, had a recent local or systemic infection, had a known malignancy (except treated basal cell skin cancer or squamous cell skin cancer for at least 5 years), had ever received treatment with any anti IL-12/23 agent, had received biological or investigational agents within the previous 3 months (or 5 drug's half-lives), had received conventional systemic psoriasis therapy or phototherapy within the previous 4 weeks, or had received topical psoriasis treatment within the previous 2 weeks. Subjects with active tuberculosis (TB) were ineligible; however those with latent TB could be enrolled if TB prophylaxis was initiated before or simultaneous to the first administration of study agent.

PHOENIX 1 comprised 766 patients, lasted 76 weeks, and was divided into 3 stages: a placebo-controlled (weeks 0-12) stage, a placebo crossover and active treatment (weeks 12-40), and a randomized withdrawal stage (weeks 40-76). ${ }^{42}$ The primary endpoint was the proportion of PASI 75 responders at week 12 . The study design allowed the investigators to assess not only the effects of this novel agent in comparison to a control group, but also long-term efficacy, evaluate possible escalation dose in partial responders, duration of the therapeutic effect after drug withdrawal, and efficacy when re-treating. Parameters selected to assess efficacy included PASI, PGA, and DLQI.

At the beginning of stage 1, subjects were randomized into 3 arms (1:1:1) to receive ustekinumab $45 \mathrm{mg}(\mathrm{n}=255)$ or $90 \mathrm{mg}(\mathrm{n}=256)$ at weeks 0 and 4 and then every 12 weeks, or placebo $(n=255)$ at weeks 0 and 4 , and then crossover to ustekinumab at week 12. In the crossover stage, patients were randomized 1:1 in the same fashion as the original treatment groups (either $45 \mathrm{mg}$ or $90 \mathrm{mg}$ ), receiving loading doses at week 12 and 16, followed by injections every 12 weeks. Then, at stage 3 (week 40), patients initially randomized to the treatment groups who had achieved PASI 75 at weeks 28 and 40 were re-randomized to either continue their therapy regimen or were withdrawn from active treatment (assigned to placebo). The objective of this withdrawal stage was to evaluate the duration of the therapeutic effect. Once these patients lost $50 \%$ of their PASI improvement, they would be re-treated (to evaluate re-treatment).

PASI scores in all groups were reevaluated at weeks 28 and 40. Those individuals considered non-responders (less than $50 \%$ improvement in their PASI scores from baseline to week 28) discontinued the study agent. Those considered partial responders (PASI scores improvements between 50\% and $74 \%$ ) were adjusted to a dosing interval of every 8 weeks. Those considered responders (PASI improvements of 75\% or more from baseline to weeks 28 and 40) were kept in the same dosing interval (every 12 weeks).

Baseline randomization was stratified by 3 different parameters, including investigational site, weight $(\leq 90 \mathrm{~kg}$ or $>90 \mathrm{~kg}$ ), and number of conventional systemic therapies to which patients had an inadequate response, intolerance, or contraindication $(<3$ or $\geq 3$ ). These led to demographics and clinical characteristics well balanced across treatment groups at baseline. Randomization at week 40 was also stratified by investigational site and baseline weight ( $\leq 90 \mathrm{~kg}$ or $>90 \mathrm{~kg}$ ).

At week 12 (primary endpoint), $67.1 \%$ of the $45 \mathrm{mg}$ group, $66.4 \%$ of the $90 \mathrm{mg}$ group, and $3.1 \%$ of the placebo group achieved PASI $75(P<0.0001$ for both treatment groups compared to placebo). Subjects receiving ustekinumab experienced a rapid onset of the clinical effects, with PASI 50 by week 2 . Other parameters such as the PGA at week 12 also showed similar clinical outcomes, with subjects achieving a "cleared or minimal" status $60.4 \%$ in the $45 \mathrm{mg}$ group, $61.7 \%$ in the $90 \mathrm{mg}$ group, and $3.9 \%$ in the placebo group $(P<0.0001$ for both treatment groups compared to placebo). Enhanced efficacy was observed throughout the active treatment phase, with maximum efficacy observed at week 24 for both dosing regimens (PASI 75 in $76.1 \%$ and $85 \%$ of the $45 \mathrm{mg}$ and $90 \mathrm{mg}$ groups respectively). Similar outcomes were obtained in subjects originally assigned to placebo, after crossing over to active treatment at week 12 . Psoriasis improvements, varying from PASI 50, PASI 75, and 
PASI 90, were seen in ustekinumab-treated patients at weeks 12 and 28, proving itself superior to placebo.

After re-randomization to maintenance/withdrawal at week 40, maintenance of PASI 75 was better among individuals receiving maintenance ustekinumab than in individuals withdrawn from treatment, up until 1 year of therapy. In the maintenance group PASI scores were steady all the way through week 76, whereas in the withdrawal group PASI scores began to progressively deteriorate by week 44 (16 weeks after withdrawal), accelerating after week 52 (24 weeks after withdrawal). The median time to loss of PASI 75 after withdrawal was about 15 weeks.

Per protocol, withdrawn patients were retreated at their original dose when they lost $50 \%$ of their baseline PASI improvement (loss of therapeutic effect). 195 patients re-initiated therapy. Among these, 85.6\% regained PASI 75 scores after 12 weeks of restarting ustekinumab.

Improvements in PASI scores were paralleled by the DLQI. DLQI scores of 0 or 1 , meaning no negative impact of psoriasis on the patients' quality of life, were achieved by $53.1 \%$ in the $45 \mathrm{mg}$ group, $52.4 \%$ in the $90 \mathrm{mg}$ group, and $6 \%$ in the placebo group at week 12 . These values were constant until the end of the study in patients receiving maintenance therapy, as opposed to the worsening reflected in the DLQI scores of patients withdrawn from ustekinumab.

The second phase III trial, PHOENIX 2, comprised of 1230 patients, lasted 52 weeks, and was divided into 3 stages: a placebo-controlled (weeks 0-12) stage, a placebo crossover and active treatment (weeks 12-28), and a randomized dose intensification stage (week $28-52) .{ }^{43}$ The primary endpoint was the proportion of PASI 75 responders at week 12. The first two stages were identical to their equivalents in PHOENIX 1 , with the exception that the second stage in this trial was shortened to 28 weeks.

Like PHOENIX 1, at the beginning of the study subjects were randomized into 3 arms $(1: 1: 1)$ to receive ustekinumab $45 \mathrm{mg}(\mathrm{n}=409)$ or $90 \mathrm{mg}(\mathrm{n}=411)$ at weeks 0 and 4 and then every 12 weeks, or placebo $(n=410)$ at weeks 0 and 4 , and then crossover to ustekinumab at week 12 . The same baseline stratification design applied in PHOENIX 1 was used in this study. In the crossover stage, patients were randomized 1:1 in the same fashion as the original treatment groups (either $45 \mathrm{mg}$ or $90 \mathrm{mg}$ ), receiving loading doses at week 12 and 16 , followed by injections every 12 weeks. Then at stage 3 (week 28), those individuals with PASI $<50$ (considered non-responders) discontinued the study agent; patients initially randomized to the treatment groups who had achieved PASI $\geq 50$ but $<75$ (partial responders), were re-randomized to either continue their therapy regimen or adjust the dosing schedule to every 8 weeks; and individuals with PASI $\geq 75$ continued on their 12-week schedule.

The second randomization at week 28 , intended for dose intensification, permitted the investigation of an objective not previously explored: the number of visits with PASI 75 between weeks 28 and 52 for the two different dosing schedule groups ( 8 vs 12 weeks). Stratification by investigational site and baseline weight ( $\leq 90 \mathrm{~kg}$ or $>90 \mathrm{~kg}$ ) was applied.

At week 12 (primary endpoint), 66.7\% of the $45 \mathrm{mg}$ group, $75.7 \%$ of the $90 \mathrm{mg}$ group, and $3.7 \%$ of those receiving placebo achieved PASI $75(P<0.0001$ for both treatment groups compared to placebo). Similarly, PGA revealed clinical outcomes with subjects achieving a "cleared or minimal" status of $68.0 \%$ in the $45 \mathrm{mg}$ group, $73.5 \%$ in the $90 \mathrm{mg}$ group, and $4.9 \%$ in the placebo group $(P<0.0001$ for both treatment groups compared to placebo). Enhanced efficacy was observed throughout the active treatment phase, with maximum efficacy observed at week 20 for both dosing regimens (PASI 75 in $74.9 \%$ and $83.5 \%$ of the $45 \mathrm{mg}$ and $90 \mathrm{mg}$ groups, respectively). Similar outcomes were obtained in placebo-assigned patients' crossing over to treatment at week 12. Like before, psoriasis improvements, varying from PASI 50, PASI 75, and PASI 90, were seen in the majority of the ustekinumab-treated patients at week 12 and 28 .

At week 28, partial responders were identified and re-randomized for dose intensification. Partial responders (22.7\% in the $45 \mathrm{mg}$ group and $15.8 \%$ in the $90 \mathrm{mg}$ group) favored higher bodyweight, more severe PGA scores, higher incidence of psoriatic arthritis, and higher likelihood of preceding failure with at least one systemic agent, in comparison to responders to 12 -weekly dosing. Predictors of partial response were narrowed to treatment with ustekinumab $45 \mathrm{mg}$, higher bodyweight, inadequate response to at least one biological agent, longer duration of psoriasis, and history of psoriatic arthritis. In addition, partial responders had lower serum drug levels at week 28 than responders, suggesting a drug level/clinical response relationship.

Dosing intensification resulted in an increased serum drug concentration. Partial responders receiving ustekinumab $45 \mathrm{mg}$ every 8 weeks did not evidence greater efficacy that those on the 12-weekly schedule, whereas with $90 \mathrm{mg}$ every 8 weeks, a greater number of visits with PASI 75 responses, and higher PASI 75 rates per se, were obtained than with 12-weekly dosing.

A sustained clinical improvement was observed through the end of the study in those individuals that reached PASI 
75 scores by week 28 . At week 52 , the median percentage improvement from baseline in PASI was $95.3 \%$ in the $45 \mathrm{mg}$ group and $95.6 \%$ in the $90 \mathrm{mg}$ group.

Promising evidence with a similar agent has been reported in a phase II trial. ABT-874 (Abbott Laboratories) is a fully human monoclonal antibody that targets IL-12/23, thus working in a similar fashion as ustekinumab. The available literature reports PASI 75 scores at week 12 above $90 \%$ (depending on the dosage evaluated) with a well tolerated safety profile. ${ }^{44}$ It is currently undergoing phase III investigations and is not yet approved by the FDA for any indication.

\section{Phase II data for psoriatic arthritis}

Psoriatic arthritis (PsA) prevalence among patients with psoriasis varies widely, and it has been estimated from $6 \%$ to $39 \% .^{5}$ Data suggest that IL-12 and IL-23 have an important role in the pathogenesis of PsA and other arthritides. $^{45,46}$

A multicenter, randomized, double-blind, placebocontrolled phase II trial evaluating the efficacy and safety of ustekinumab for PsA was recently published. ${ }^{47}$ Although detailed description of this trial goes beyond the scope of this review, is crucial to highlight the overall study design and results. 146 patients with active PsA underwent 1:1 randomization to receive ustekinumab or placebo. Subjects assigned to the ustekinumab group received $63 \mathrm{mg}$ or $90 \mathrm{mg}$ subcutaneously (SC) weekly for 4 weeks (weeks 0 to 3 ) followed by placebo injections on weeks 12 and 16; patients assigned to the control group received placebo injections at weeks 0 to 3, followed by ustekinumab $63 \mathrm{mg} \mathrm{SC}$ at weeks 12 and 16. A filtration procedure implemented during dose preparation led to the dose change from $90 \mathrm{mg}$ to $63 \mathrm{mg}$. The primary endpoint was American College of Rheumatology (ACR) 20 response at week 12. Participants were followed up to week 36.

Men and women aged 18 years or older were eligible if they had active PsA (defined as 3 or more swollen joints and 3 or more tender joints, and either C-reactive protein (CRP) of $15 \mathrm{mg} / \mathrm{L}$ or greater or morning stiffness for at least $45 \mathrm{~min}$ ) for at least 6 months. Patients also had to have active plaque psoriasis, and an unsatisfactory response to DMARDs, NSAIDs, anti-TNF agents, or a combination of these. If subjects were on a stable course of therapy of MTX (up to $25 \mathrm{mg} /$ week), corticosteroids (up to $10 \mathrm{mg}$ /day of prednisone or equivalent), NSAIDs, or a combination of these drugs, they were allowed to continue their regimen. Patients were ineligible if they had received biological or investigational agents within the previous 3 months, had received conventional systemic psoriasis therapy or phototherapy within the previous 4 weeks, or had received topical psoriasis treatment within the previous 2 weeks.

Parameters selected to assess efficacy included ACR criteria, Disease Activity Index score (DAS) 28, PASI, PGA, DLQI, and Health Assessment Questionnaire (HAQ) disability index. ACR score assesses multiple disease criteria, and evaluates percentage improvement of 20\%, 50\% and 70\% (ACR 20, ACR 50, and ACR 70) from baseline. DAS 28 is a derived index that combines tender and swollen joint counts $(\mathrm{n}=28)$, CRP, and patient's global assessment of disease activity.

Efficacy assessed at week 12 (primary endpoint) revealed ACR 20 scores for the active treatment group of 42\% (32 of 76 ), as compared with $14 \%$ (10 of 70 ) in the control group $(P=0.0002)$. Also, more patients in the ustekinumab group achieved ACR 50 (25\% [19/76] vs placebo 7\% [5/70]) and ACR 70 (11\% [8/76] vs placebo 0) responses at week 12. All ACR responses in the active treatment group peaked at weeks 16 to 20, and then decreased slowly to week 36 . After placebo patients crossed over to ustekinumab at week 12, they achieved similar ACR 20 responses to those in the treatment group at week $24(51 \%, 28 / 55)$, week 28 (45\%, $24 / 53)$, and at week $36(42 \%, 21 / 50)$. Clinical responses were supported by the substantial improvements observed in the DLQI and HAQ disability index at week 12 in the treatment group. Psoriasis measures were also reduced with ustekinumab treatment.

Results from this trial show that ustekinumab is efficacious for the treatment of active PsA. Larger and longer studies are needed to further characterize ustekinumab for the treatment of this condition.

\section{Safety and tolerability}

Unlike efficacy data (analyzed based on assigned treatment group), safety data were analyzed based on actual treatment and included all patients who had received at least one dose of ustekinumab. ${ }^{42,43}$ Since both PHOENIX 1 and 2 reported similar safety data, when possible, they are presented together (safety data from the phase II PsA trial were not included in this section).

Ustekinumab was generally well tolerated at both doses (45 and $90 \mathrm{mg}$ ) and dosing schedules (every 8 and 12 weeks). The majority of adverse events were mild, nonserious and did not require treatment adjustment. The rate and types of adverse events were much the same across all groups (treatment and placebo) throughout the different phases of both 
studies, although absolute event rates differed across study phases consistent with different lengths of follow up. An exception to this occurred during the dose intensification phase in PHOENIX 2, where adverse events were more frequent in patients receiving treatment every 8 weeks $(72.7 \%)$ than in those receiving treatment every 12 weeks $(63 \%)$. Paradoxically, serious adverse events were more frequent in the 12 -weekly (7.4\%) than in the 8-weekly group (2.6\%). By the end of the trials, no dose response was seen in the rates of adverse events, serious events, or events leading to study agent discontinuation. The most commonly reported adverse events were upper respiratory tract infections, nasopharyngitis, headaches, and arthralgias. The most common serious events included infections, malignancies, and cardiovascular events. $^{42,43}$

Rates of laboratory abnormalities were low and generally similar between treatment groups and placebo groups. Ustekinumab showed no effect on glucose, hemoglobin A1c, neutrophil counts, or D-dimer levels. Antibodies to ustekinumab were found in $5.1 \%$ of the patients by the end of week 76 (PHOENIX 1), and in $5.4 \%$ by the end of week 52 (PHOENIX 2). They were mostly low titer. Injection site reactions were infrequent ( $1 \%$ for ustekinumab, and $0.4 \%$ for placebo), probably due to the scarcity of the injections. No anaphylactic or serum-sickness-like reactions were reported.

\section{Economic evidence and resource utilization}

There are no studies yet that include an economic evaluation of ustekinumab in psoriasis. The drug was recently approved in Canada (December 2008) and in Europe by the European Medicines Agency (EMEA) (January 16, 2009). More recently, the drug was approved by the FDA for patients with moderate to severe forms of psoriasis (September 25, 2009).

Because this drug represents the first agent developed among its class, it's difficult to predict its overall impact. Ustekinumab's clinical trials have shown an apparent better risk/benefit ratio that, if supported by future more powerful data, may possibly propel this novel agent to become an even more attractive therapeutic alternative. Its infrequent dosing schedule, and the possibility of added benefit for patients with concomitant psoriatic arthritis, further increase the potential of the drug. It will be important to determine in future studies the drug's effect on reductions in direct and indirect costs of the disease, such as visits to physicians, work days lost to the disease or treatments, etc.

\section{Patient group/population}

Ustekinumab trials have shown efficacy in men and women, aged 18 years or older, with a diagnosis of chronic moderate to severe plaque psoriasis. Due to the profile of the patients that participated in the phase III trials (eg, elevated bodyweight, failed prior systemic therapies), it seems that this drug could potentially benefit patients with recalcitrant psoriasis who have failed previous therapeutic regimens, including conventional and biologic agents. In addition, patients with concomitant psoriatic arthritis may benefit from it as well.

Intermittently, patients with refractory psoriasis have demanded from their treating physicians trials of different drugs, combining and rotating agents arbitrarily in the search of a reasonable improvement. Although not generally resulting in satisfactory clinical outcomes, toxicities and side effects can arise, further complicating the picture. An agent with efficacy rates as promising as ustekinumab's might represent a valuable alternative for these challenging cases. What is more, so far its safety profile has shown to be relatively benign.

\section{Clinical potential}

At the primary endpoint (week 12), ustekinumab has successfully established its superiority over placebo and over currently available psoriasis therapies. Not only have more patients achieved PASI 75 scores than with other biologics, but also its rapid onset of action and better dosing profile makes this agent a potentially valuable alternative for psoriatic patients. Moreover, the duration of the phase III trials demonstrated ustekinumab's sustained long-term clinical responses (up to 76 weeks of treatment). All analyses were done using NRI, yielding reliable, if not underestimated, efficacy rates. ${ }^{48}$

Patients with refractory psoriasis that have failed to respond to other systemic and/or biologic agents seem to be the kind of cases that could potentially benefit most from this novel compound. Once additional data become available, the target population for this drug will be furthered defined. Because of its convenient dosing schedule as an infrequent monotherapeutic agent, it may translate into lower costs and better chance for compliance.

Additional investigations evaluating long-term efficacy, and more specifically safety data, are needed before ustekinumab can become a major player in the biologics market. Assessment of clinical effects on psoriatic arthritis might be part of the objectives in future ustekinumab studies. 


\section{Disclosure}

Dr Gordon has received honoraria and research support from Centocor, the manufacturers of ustekinumab.

\section{References}

1. Schon MP, Boehncke WH. Psoriasis. N Engl J Med. 2005;352: 1899-1912.

2. van de Kerkhof PC. Consistent control of psoriasis by continuous long-term therapy: the promise of biological treatments. $J$ Eur Acad Dermatol Venereol. 2006;20:639-650.

3. Zheng Y, Danilenko DM, Valdez P, et al. Interleukin-22, a $\mathrm{T}(\mathrm{H}) 17$ cytokine, mediates IL-23-induced dermal inflammation and acanthosis. Nature. 2007;445:648-651.

4. Torti DC, Feldman SR. Interleukin-12, interleukin-23, and psoriasis: current prospects. J Am Acad Dermatol. 2007;57:1059-1068.

5. Gelfand JM, Gladman DD, Mease PJ, et al. Epidemiology of psoriatic arthritis in the population of the United States. J Am Acad Dermatol. 2005;53:573-577.

6. Gisondi P, Tessari G, Conti A, et al. Prevalence of metabolic syndrome in patients with psoriasis: a hospital-based case-control study. $\mathrm{Br} \mathrm{J}$ Dermatol. 2007;157:68-73.

7. Chen YJ, Wu CY, Shen JL, et al. Psoriasis independently associated with hyperleptinemia contributing to metabolic syndrome. Arch Dermatol. 2008;144:1571-1575.

8. Nijsten T, Wakkee M. Complexity of the association of between psoriasis and comorbidities. J Invest Dermatol. 2009;129:1601-1603.

9. Gupta MA, Gupta AK. Depresion and suicidal ideation in dermatology patients with acne, alopecia areata, atopic dermatitis and psoriasis. Br J Dermatol. 1998;139:846-850.

10. Russo PA, Ilchef R, Cooper AJ. Psychiatric morbidity in psoriasis: a review. Australas J Dermatol. 2004;45:155-159; quiz 160-161.

11. Magin PJ, Pond CD, Smith WT, Watson AB, Goode SM. A cross-sectional study of psychological morbidity in patients with acne, psoriasis and atopic dermatitis in specialist dermatology and general practices. J Eur Acad Dermatol Venereol. 2008;22: $1435-1444$.

12. Krueger G, Koo J, Lebwohl M, Menter A, Stern RS, Rolstad T. The impact of psoriasis on quality of life: results of a 1998 National Psoriasis Foundation patient-membership survey. Arch Dermatol. 2001;137:280-284.

13. Smith CH, Anstey AV, Barker JN, et al. British Association of Dermatologists guidelines for use of biological interventions in psoriasis 2005 . Br J Dermatol. 2005;153:486-497.

14. Rapp SR, Feldman SR, Exum ML, Fleischer AB Jr, Reboussin DM. Psoriasis causes as much disability as other major medical diseases. $J$ Am Acad Dermatol. 1999;41:401-407.

15. Nair RP, Stuart PE, Nistor I, et al. Sequence and haplotype analysis supports HLA-C as the psoriasis susceptibility 1 gene. Am J Hum Genet 2006;78:827-851.

16. Menter A, Gottlieb A, Feldman SR, et al. Guidelines of care for the management of psoriasis and psoriatic arthritis: Section 1. Overview of psoriasis and guidelines of care for the treatment of psoriasis with biologics. $J$ Am Acad Dermatol. 2008;58: $826-850$

17. Krueger G, Ellis CN. Psoriasis - recent advances in understanding its pathogenesis and treatment. J Am Acad Dermatol. 2005;53:S94-S100.

18. Somani AK, Yang MF, Cooper KD, McCormick TS. Cytokines and psoriasis. When cytokines become "pathokines". G Ital Dermatol Venereol. 2007;142:679-690.

19. Nickoloff BJ. Cracking the cytokine code in psoriasis. Nat Med. 2007;13:242-244.

20. Reddy M, Davis C, Wong J, Marsters P, Pendley C, Prabhakar U. Modulation of CLA, IL-12R, CD40L, and IL-2Ralpha expression and inhibition of IL-12- and IL-23-induced cytokine secretion by CNTO 1275. Cell Immunol. 2007;247:1-11.
21. Zaba LC, Cardinale I, Gilleaudeau P, et al. Amelioration of epidermal hyperplasia by TNF inhibition is associated with reduced Th17 responses. J Exp Med. 2007;204:3183-3194.

22. Guttman-Yassky E, Lowes MA, Fuentes-Duculan J, et al. Major differences in inflammatory dendritic cells and their products distinguish atopic dermatitis from psoriasis. J Allergy Clin Immunol. 2007;119:1210-1217.

23. Zaba LC, Krueger JG, Lowes MA. Resident and "inflammatory" dendritic cells in human skin. J Invest Dermatol. 2009;129:302-308.

24. Nickoloff BJ, Qin JZ, Nestle FO. Immunopathogenesis of psoriasis. Clin Rev Allergy Immunol. 2007;33:45-56.

25. Nickoloff BJ, Xin H, Nestle FO, Qin JZ. The cytokine and chemokine network in psoriasis. Clin Dermatol. 2007;25:568-573.

26. Toichi E, Torres G, McCormick TS, et al. An anti-IL-12p40 antibody down-regulates type 1 cytokines, chemokines, and IL-12/IL-23 in psoriasis. J Immunol. 2006;177:4917-4926.

27. Nair RP, Ruether A, Stuart PE, et al. Polymorphisms of the IL12B and IL23R genes are associated with psoriasis. $J$ Invest Dermatol. 2008;128:1653-1661.

28. Henseler T, Schmitt-Rau K. A comparison between BSA, PASI, PLASI and SAPASI as measures of disease severity and improvement by therapy in patients with psoriasis. Int J Dermatol. 2008;47:1019-1023.

29. Gordon KB. Clinical Outcomes Measurements. In Gordon KB, Ruderman EM, eds. Psoriasis and Psoriatic Arthritis, An Integral Approach. New York: Springer; 2005:125-128.

30. Langley RG, Ellis CN. Evaluating psoriasis with Psoriasis Area and Severity Index, Psoriasis Global Assessment, and Lattice System Physician's Global Assessment. J Am Acad Dermatol. 2004;51:563-569.

31. Menter A, Griffiths CE. Current and future management of psoriasis. Lancet. 2007;370:272-284.

32. Strowd LC, Yentzer BA, Fleischer AB Jr, Feldman SR. Increasing use of more potent treatments for psoriasis. J Am Acad Dermatol. 2008:60:478-481.

33. Feldman SR, Horn EJ, Balkrishnan R, et al. Psoriasis: improving adherence to topical therapy. J Am Acad Dermatol. 2008;59:1009-1016.

34. Nijsten TE, Stern RS. The increased risk of skin cancer is persistent after discontinuation of psoralen+ultraviolet A: a cohort study. J Invest Dermatol. 2003;121:252-258.

35. Strober BE, Siu K, Menon K. Conventional systemic agents for psoriasis. A systematic review. $J$ Rheumatol. 2006;33:1442-1446.

36. Gottlieb A, Korman NJ, Gordon KB, et al. Guidelines of care for the management of psoriasis and psoriatic arthritis: Section 2. Psoriatic arthritis: overview and guidelines of care for treatment with an emphasis on the biologics. J Am Acad Dermatol. 2008;58:851-864.

37. FDA Medwatch for Efalizumab. Feb 2009. URL: www.fda.gov

38. Lebwohl M, Menter A, Koo J, Feldman SR. Combination therapy to treat moderate to severe psoriasis. J Am Acad Dermatol. 2004;50: 416-430.

39. Nijsten T, Margolis DJ, Feldman SR, Rolstad T, Stern RS. Traditional systemic treatments have not fully met the needs of psoriasis patients: results from a national survey. J Am Acad Dermatol. 2005;52: 434-444.

40. Papp KA. The long-term efficacy and safety of new biological therapies for psoriasis. Arch Dermatol Res. 2006;298:7-15.

41. Krueger GG, Langley RG, Leonardi C, et al. A human interleukin-12/23 monoclonal antibody for the treatment of psoriasis. $N$ Engl $J$ Med. 2007;356:580-592.

42. Leonardi CL, Kimball AB, Papp KA, et al. Efficacy and safety of ustekinumab, a human interleukin-12/23 monoclonal antibody, in patients with psoriasis: 76-week results from a randomised, doubleblind, placebo-controlled trial (PHOENIX 1). Lancet. 2008;371: 1665-1674.

43. Papp KA, Langley RG, Lebwohl M, et al. Efficacy and safety of ustekinumab, a human interleukin-12/23 monoclonal antibody, in patients with psoriasis: 52-week results from a randomised, doubleblind, placebo-controlled trial (PHOENIX 2). Lancet. 2008;371: $1675-1684$. 
44. Kimball AB, Gordon KB, Langley RG, Menter A, Chartash EK, Valdes J. Safety and efficacy of ABT-874, a fully human interleukin $12 / 23$ monoclonal antibody, in the treatment of moderate to severe chronic plaque psoriasis: results of a randomized, placebo-controlled, phase 2 trial. Arch Dermatol. 2008;144:200-207.

45. Kim M, Min S, Cho M, et al. The role of IL-12 in inflammatory activity of patients with rheumatoid arthritis (RA). Clin Exp Immunol. 2000;119:175-181.

46. Szodoray P, Alex P, Chapell-Woodward CM, et al. Circulating cytokines in Norwegian patients with psoriatic arthritis determined by a multiple cytokine array system. Rheumatology. 2007;46:417-425.

47. Gottlieb A, Menter A, Mendelsohn A, et al. Ustekinumab, a human interleukin 12/23 monoclonal antibody, for psoriatic arthritis: Randomised, double-blind, placebo-controlled, crossover trial. Lancet. 2009;373:633-40.

48. Heritier SR, Gebski VJ, Keech AC. Inclusion of patients in clinical trial analysis: the intention-to-treat principle. Med J Aust. 2003;179:438-440.

49. Tzu J, Krulig E, Cardenas V, Kerdel FA. Biological agents in the treatment of psoriasis. G Ital Dermatol Venereol. 2008;143:315-327.

50. Brimhall AK, King LN, Licciardone JC, Jacobe H, Menter A. Safety and efficacy of alefacept, efalizumab, etanercept and infliximab in treating moderate to severe plaque psoriasis: a meta-analysis of randomized controlled trials. Br J Dermatol. 2008;159:274-285.
51. Lebwohl M, Christophers E, Langley R, Ortonne JP, Roberts J, Griffiths CE. An international, randomized, double-blind, placebocontrolled phase 3 trial of intramuscular alefacept in patients with chronic plaque psoriasis. Arch Dermatol. 2003;139:719-727.

52. Gordon KB, Langley RG, Leonardi C, et al. Clinical response to adalimumab treatment in patients with moderate to severe psoriasis: double-blind, randomized controlled trial and open-label extension study. J Am Acad Dermatol. 2006;55:598-606.

53. Menter A, Tyring SK, Gordon K, et al. Adalimumab therapy for moderate to severe psoriasis: A randomized, controlled phase III trial. J Am Acad Dermatol. 2008;58:106-115.

54. Papp KA, Tyring S, Lahfa M, et al. A global phase III randomized controlled trial of etanercept in psoriasis: safety, efficacy, and effect of dose reduction. Br J Dermatol. 2005;152:1304-1312.

55. Tyring S, Gottlieb A, Papp K, et al. Etanercept and clinical outcomes, fatigue, and depression in psoriasis: double-blind placebo-controlled randomised phase III trial. Lancet. 2006;367:29-35.

56. Reich K, Nestle FO, Papp K, et al. Infliximab induction and maintenance therapy for moderate-to-severe psoriasis: a phase III, multicentre, double-blind trial. Lancet. 2005;366:1367-1374.

57. Hamilton CD. Infectious complications of treatment with biologic agents. Curr Opin Rheumatol. 2004;16:393-398.
Core Evidence

\section{Publish your work in this journal}

Core Evidence is an international, peer-reviewed open-access journal evaluating the evidence underlying the potential place in therapy of drugs throughout their development lifecycle from preclinical to postlaunch. The focus of each review is to evaluate the case for a new drug or class in outcome terms in specific indications and patient groups.

\section{Dovepress}

The manuscript management system is completely online and includes a very quick and fair peer-review system, which is all easy to use. Visit http://www.dovepress.com/testimonials.php to read real quotes from published authors. 